See discussions, stats, and author profiles for this publication at: https://www.researchgate.net/publication/257264946

Paracoccidioidomicose pulmonar: relato de caso clínico com aspetos em tomografia computorizada de alta resolução

Article in Revista portuguesa de pneumologia · July 2012

Dol: 10.1016/j.rppneu.2012.02.001

CITATIONS

3

5 authors, including:

Monica Armas

Serviço de Saúde da RAM, E.P.E.

6 PUBLICATIONS 8 CITATIONS

SEE PROFILE

Rubina Alves

Universitat Internacional de Catalunya

48 PUBLICATIONS 189 CITATIONS

SEE PROFILE

Some of the authors of this publication are also working on these related projects:

Doctoral Thesis: PRP View project

Chapter of Book View project
169

Catarina Ruivo

17 PUBLICATIONS 48 CITATIONS

SEE PROFILE 


\title{
Paracoccidioidomicose pulmonar: relato de caso clínico com aspetos em tomografia computorizada de alta resolução
}

\author{
M. Armas ${ }^{a, *}$, C. Ruivo ${ }^{b}$, R. Alves ${ }^{c}, M$. Gonçalves $^{a}$ e L. Teixeira ${ }^{b}$ \\ a Serviço de Radiologia, Hospital Central do Funchal, Funchal, Portugal \\ b Serviço de Radiologia, Hospitais da Universidade de Coimbra, Coimbra, Portugal \\ c Serviço de Dermatologia, Hospital Central do Funchal, Funchal, Portugal
}

Recebido a 11 de dezembro de 2011; aceite a 4 de janeiro de 2012

Disponível na Internet a 22 de março de 2012

\section{PALAVRAS-CHAVE Paracoccidioidomi- cose; Paracoccidioides brasiliensis; Fibrose pulmonar; Tomografia computorizada de alta resolução}

\section{KEYWORDS} Paracoccidioidomycosis; Paracoccidioides brasiliensis; Pulmonary fibrosis; High-resolution computed tomography

\begin{abstract}
Resumo A Paracoccidioidomicose é uma micose sistémica endémica nas áreas rurais da América Latina, uma fonte importante de imigrantes e destino de emigração e turismo europeu, a maioria dos casos ocorrendo no Brasil, Argentina, Venezuela e Colômbia. Os autores descrevem o caso clínico de um paciente com 43 anos, anteriormente emigrado na Venezuela e residente em Portugal há 8 anos, que se apresenta com lesão cutânea isolada. Embora sem queixas relevantes do foro respiratório, apresentava extensas lesões do parênquima pulmonar caracterizadas por tomografia computorizada de alta resolução (TCAR). Foi realizada biópsia da lesão cutânea e exame micológico da expetoração que revelaram infeção por Paracoccidioides brasiliensis (PB).

(C) 2011 Sociedade Portuguesa de Pneumologia. Publicado por Elsevier España, S.L. Todos os direitos reservados.
\end{abstract}

\section{Pulmonary paracoccidioidomycosis: A case report with high-resolution computed tomography findings}

\begin{abstract}
Paracoccidioidomycosis is a systemic mycosis which is endemic in rural areas of Latin America, an important European source of immigrants and a growing European touristic destination as well, with most cases occurring in Brazil, Argentina, Venezuela and Colombia. The authors report a case of a 43 year old man who previously worked in Venezuela and is living in Portugal for 8 years, presenting with a single cutaneous lesion. Despite the absence of valuable respiratory complaints, severe lung damage was found with high-resolution computed tomography (HRCT). Biopsy of the cutaneous lesion and mycologic sputum examination were performed revealing Paracoccidioides brasiliensis infection.

(C) 2011 Sociedade Portuguesa de Pneumologia. Published by Elsevier España, S.L. All rights reserved.
\end{abstract}

\footnotetext{
* Autor para correspondência.

Correio eletrónico: mncarmas@yahoo.com (M. Armas).
}

0873-2159/\$ - see front matter @ 2011 Sociedade Portuguesa de Pneumologia. Publicado por Elsevier España, S.L. Todos os direitos reservados. doi:10.1016/j.rppneu.2012.02.001 


\section{Introdução}

A Paracoccidioidomicose (PCM) é a micose sistémica endémica mais frequente da América Latina, atingindo cerca de $10 \%$ da população em regiões subtropicais do Brasil ${ }^{1}$, afetando sobretudo a população agrícola, com maior incidência entre os 25 e 60 anos $^{2}$. 0 agente causal, o Paracoccidioides brasiliensis (PB), é um fungo dimórfico que pode permanecer viável no hospedeiro por longos períodos, com reativação da doença até vários anos após a infeção inicial. A porta de entrada é o aparelho respiratório, sendo o pulmão o órgão mais afetado ${ }^{1}$. A infeção crónica com doença pulmonar severa e evolução para fibrose terminal pode ocorrer mesmo na ausência de sintomatologia pronunciada. A disseminação a partir de uma lesão pulmonar primária pode afetar outros órgãos, mais frequentemente a pele e membranas mucosas, mas também as glândulas suprarrenais, rins, aparelho gastrointestinal, fígado, baço e o sistema nervoso central ${ }^{3,4}$. As sequelas mais comuns desta micose incluem fibrose com insuficiência respiratória, cor pulmonale e também doença de Addison e má absorção intestinal ${ }^{2}$. Os autores apresentam um caso de PCM com envolvimento pulmonar e cutâneo.

\section{Caso clínico}

Indivíduo português do sexo masculino com 43 anos de idade, trabalhador agrícola na Venezuela até 2001, surge com lesão cutânea ulcerada localizada no dorso, com 2 meses de evolução (fig. 1). Não apresentava outras queixas nem antecedentes patológicos relevantes, excetuando lesão ulcerada pruriginosa e indolor na mucosa nasal em 1996, para a qual efetuou durante 6 meses tratamento que não soube especificar. Apresentava bom estado geral, sem febre, adenomegálias palpáveis ou hepatoesplenomegália. O estudo analítico não revelou alterações, com negatividade nomeadamente para o HIV 1 e 2 . Na auscultação pulmonar apresentava alterações bilaterais difusas, pelo que efetuou uma radiografia torácica que mostrou um padrão

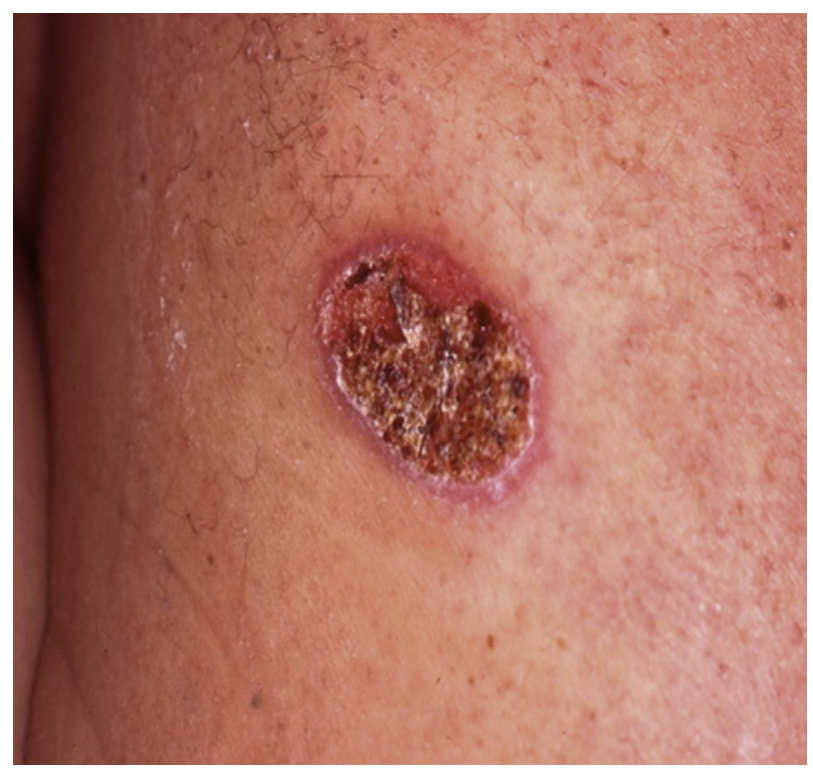

Figura 1 Lesão ulcerada no dorso com 2 meses de evolução. reticulo-nodular bilateral predominando nos andares médios e superiores, associado a consolidação segmentar na base direita.

O estudo pulmonar por TCAR revelou exuberantes lesões bilaterais do parênquima pulmonar, coexistindo alterações fibrosantes com aspetos de doença inflamatória ativa, particularmente nos lobos médio e superiores, apesar da escassa sintomatologia respiratória. Identificaram-se múltiplas lesões nodulares espiculadas e irregulares, com dimensões entre 8 e $25 \mathrm{~mm}$, algumas com cavitação central e confluentes, mais proeminentes nos lobos superiores. Outras alterações incluíam uma área de consolidação com broncograma aéreo no lobo médio, distorção arquitetural, focos dispersos com atenuação em «vidro despolido», espessamento de septos interlobulares, bandas de parênquima, espessamento pleural espiculado difuso, bolhas de enfisema apicais e bronquiectasias de tração. Observaram-se igualmente adenopatias hilares bilaterais e ectasia irregular da traqueia (fig. 2).

A biópsia da lesão cutânea e o exame micológico da expetoração revelaram infeção por PB.

Foi instituída terapêutica com itraconazole $(200 \mathrm{mg} / \mathrm{d}$ durante 2 meses, seguido de $100 \mathrm{mg} / \mathrm{d}$ durante 8 meses), com evolução favorável do quadro clínico.

\section{Discussão}

A PCM foi descrita inicialmente pelo médico brasileiro Lutz, em $1908^{2}$. É a micose endémica mais frequente na América Latina. O Brasil lidera em número de casos, com especial prevalência em regiões subtropicais onde afeta cerca de $10 \%$ da população.

É uma doença granulomatosa sistémica, predominando as formas pulmonares e cutâneo-mucosas. Os pulmões estão comprometidos em 50 a $100 \%$ dos casos $^{1}$. A via principal de infeção é a respiratória, por inalação dos esporos do PB presentes no solo ${ }^{1,2,4,7,8}$. O contacto inicial do hospedeiro com o fungo habitualmente evolui para uma infeção subclínica, com formação inicial de granuloma pulmonar, que poderá ter características semelhantes ao complexo primário da tuberculose ${ }^{1,2}$.

$\mathrm{Na}$ maioria dos indivíduos os mecanismos naturais de defesa permitem estabelecer um equilíbrio entre o hospedeiro e o agente, permanecendo o fungo viável na forma latente ${ }^{1,3,4,6}$. Entretanto, a doença pode progredir a partir do foco primário pulmonar, mais frequentemente para a pele e mucosas da via aérea e cavidade oral, com formação de lesões granulomatosas ulceradas hemorrágicas ${ }^{5}$.

Duas formas clínicas foram descritas:

- uma forma aguda/subaguda ou do tipo juvenil que surge em menos de $10 \%$ dos casos, afetando ambos os sexos em idade inferior a 25 anos, com febre, emagrecimento e alteração do estado geral. Há rápido e progressivo envolvimento de órgãos com adenomegalias difusas, superficiais e profundas, e hepatoesplenomegalia, podendo coexistir com menor frequência lesões cutâneas e osteolíticas. O envolvimento do intestino delgado ocorre em cerca de $50 \%$ dos casos. Raramente existe envolvimento do pulmão e medula óssea. As complicações mais comuns são a obstrução linfática, 

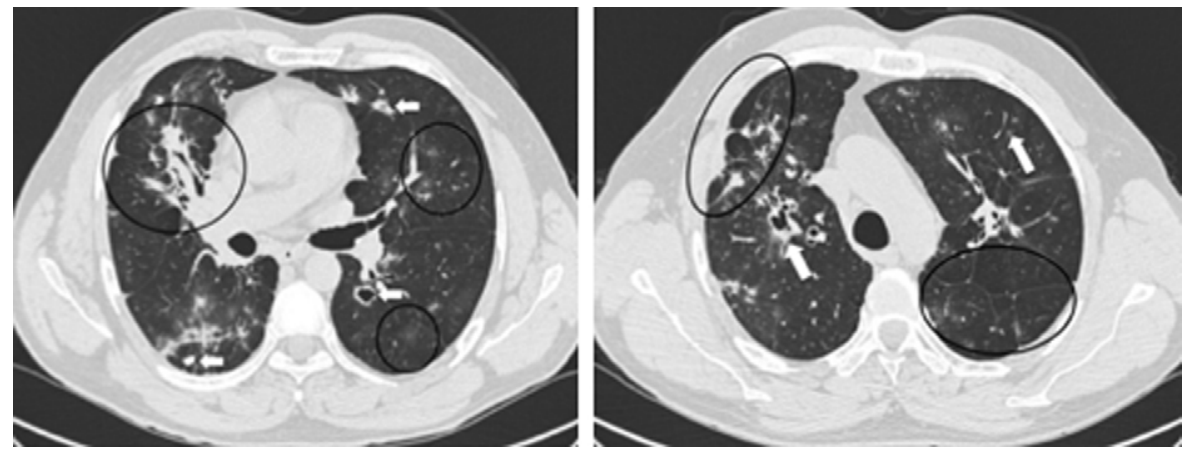

Figura 2 Aspetos em TCAR pulmonar.

Esquerda: Área de consolidaçãono no lobo médio com broncograma aéreo e distorção arquitectural. Áreas de padrão em «vidro despolido» dispersas no campo pulmonar esquerdo. Granulomas bilaterais, alguns com cavitação (setas). Espessamento espiculado da pleura mediastínica.

Direita: Enfisema paracicatricial e cavitação no lobo superior direito. Banda de parênquima (seta) e espessamento de septos interlobulares no parênquima pulmonar esquerdo.

má absorção intestinal ou enteropatia perdedora de proteínas $^{2,4}$.

- uma forma crónica que surge sobretudo no adulto do sexo masculino (relação homem / mulher de cerca de 10:1 a 25:1), dos 25 aos 60 anos, em que o órgão mais frequentemente afetado é o pulmão, seguido da pele e membranas mucosas. Os doentes podem encontrarse assintomáticos ou referirem dispneia, tosse e raramente hemoptises. A febre é rara e o exame físico é frequentemente normal. Pode ser paradoxal a pobreza do quadro clínico respiratório quando comparado à exuberância das lesões pulmonares. A morbilidade nestes casos relaciona-se sobretudo com o desenvolvimento de insuficiência respiratória e cor pulmonale $e^{1,2,4,6}$. Raramente pode manifestar-se com um quadro de pneumotórax espontâneo ${ }^{6}$.

A radiografia do tórax na infeção por $\mathrm{PB}$ pode apresentar diversas alterações: opacidades lineares e reticulares, nódulos com dimensões variáveis, infiltrados mal definidos, áreas de consolidação do espaço aéreo e cavitação ${ }^{6}$. Este exame apresenta, porém, limitada capacidade na avaliação de doenças pulmonares difusas. Deste modo, a TCAR tornouse o método de escolha na avaliação dos pacientes com suspeita clínica e/ou laboratorial de PCM pulmonar.

As alterações do parênquima pulmonar têm habitualmente uma distribuição bilateral, praticamente simétrica e, embora afetando todo o pulmão, predominam nos lobos médio, lingular e superiores ${ }^{7-10}$. Podem incluir simultaneamente lesões em fase ativa e alterações crónicas e fibrosantes.

A doença pulmonar manifesta-se em TCAR por áreas de consolidação, opacidades em «vidro despolido», nódulos parenquimatosos dispersos (granulomas ou paracoccidiomas), espessamento de septos interlobulares, espessamento pleural espiculado, espessamento de paredes brônquicas, bronquiectasias de tração, dilatação traqueal, distorção arquitetural, bandas parenquimatosas e enfisema paracicatricial $^{7-10}$. Adenopatias hilares e mediastínicas estão frequentemente presentes ${ }^{9,10}$.
A consolidação do parênquima traduz uma pneumonia focal, que se inicia habitualmente por uma alveolite descamativa (associada a opacidades em «vidro despolido») e que consiste microscopicamente num infiltrado inflamatório rico em fungos ${ }^{8}$. 0 sinal do «halo invertido» (definido como uma atenuação em «vidro despolido» central circundada por consolidação periférica densa em forma de crescente ou anel), surge em cerca de $10 \%$ dos doentes com infeção activa $^{11,12}$.

Os granulomas caracterizam-se por dimensões variáveis e contornos irregulares, sobretudo espiculados, alguns adquirindo formas bizarras e com tendência à confluência. A cavitação é frequente, traduzindo um processo inflamatório do tipo exsudativo ${ }^{9,10}$.

A fibrose pulmonar é um achado frequente com espessamento dos septos inter e intralobulares, espessamento das paredes brônquicas, distorção arquitetural, bandas parenquimatosas, reticulado intralobular e espessamento irregular do interstício axial peri-hilar, envolvendo gânglios linfáticos hilares, brônquios principais e ramos da artéria pulmonar. Este aspeto pode ser responsável pelo desenvolvimento de cor pulmonale $e^{1,7-10}$.

0 diagnóstico definitivo desta micose é obtido pela demonstração do PB em fluídos biológicos ou tecidos, principalmente por exame micológico direto e/ou histopatológico, ou por técnicas serológicas (títulos de anticorpos específicos) que têm igualmente utilidade na avaliação da resposta ao tratamento e deteção de recidiva ${ }^{2,4,5}$.

0 tratamento da doença clínica ativa pode ser feito com trimetoprim-sulfometoxazol ou itraconazole. Os casos mais graves de doença crónica ou aguda podem ser tratados com anfotericina $B^{2,12,13}$.

\section{Conclusões}

Embora pouco frequente na nossa prática clínica diária, num contexto adequado de exposição a zonas endémicas, mesmo não recente, a PCM deve ser considerada como hipótese diagnóstica. A TCAR é um meio de diagnóstico essencial no estudo destes doentes, permitindo a correta avaliação do grau de compromisso do parênquima pulmonar. 


\section{Conflito de interesses}

Os autores declaram não haver conflito de interesses.

\section{Bibliografia}

1. Souza Jr A, Gasparetto E, Davaus T, Escuissato D, Marchiori E. High-Resolution CT Findings of 77 Patients with Untreated Pulmonary Paracoccidioidomycosis. AJR. 2006;187:1248-52.

2. Carvalho R, Branquinho F, Theias R, Perloiro M. Paracoccidiodomicose Brasiliensis: a propósito de um caso clínico. Rev Soc Port Med Int. 2009;16:170-2.

3. Gasparetto EL, Liu CB, Carvalho Neto A, Rogacheski E. Central nervous system paracoccidioidomycosis: imaging findings in 17 cases. J Comput Assist Tomogr. 2003;27:12-21.

4. Brummer E, Castaneda E, Restropo A. Paracoccidioidomycosis: an Update. Clin Microb Rev Apr. 1993;6:89-117.

5. Negroni R. Paracoccidioidomycosis (South American Blastomycosis, Lutz's Mycosis). Int J Dermatol. 1993;32:847-59.

6. Pereira M, Marchiori E, Zanetti G, Abdalla G, Ventura N, Constantino $\mathrm{C}$, et al. Spontaneous pneumothorax as an atypical presentation of pulmonary paracoccidioidomycosis: a case report with emphasis on the imaging findings. Case Report Med. 2010;2010:961984. Epub 2010 Jun 20. doi: $10.1155 / 2010 / 961984$.
7. Marchiori E, Moraes HP, Muniz MAS, Santos MLO, Capone D. Paracoccidioidomicose: correlação da tomografia computadorizada de alta resolução com a anatomopatologia. Radiol Bras. 2000;33:333-40.

8. Marchiori E, Valiante PM, Escuissato DL, Souza Jr AS, Mano CM, Zanetti G, et al. Paracoccidioidomycosis: HighResolution Computed Tomography - Pathologic Correlation. Eur J Radiol. 2011;77:80-4.

9. Muniz M, Marchiori E, Magnago M, Moreira L, Almeida Júnior J. Paracoccidioidomicose pulmonar - aspectos na tomografia computorizada de alta resolução. Radiol Bras. 2002;35: 147-54.

10. Funari M, Kavakama J, Shikanai-Yasuda MA, Castro LG, Bernard G, Rocha MS, et al. Chronic pulmonary paracoccidioidomycosis (South American blastomycosis): high-resolution CT findings in 41 patients. AJR. 1999;173:59-64.

11. Gasparetto EL, Escuissato DL, Davaus T, De Cerqueira EM, Souza Jr AS, Marchiori E, et al. Reversed halo sign in pulmonary paracoccidioidomycosis. Am J Roentgenol. 2005;184: 1932-4.

12. Freitas Filho M, Gonçalves F, Basílio M, Mançano A, Cherulli B, Barreiros M. Paracoccidioidomicose pulmonar: relato de dois casos enfantizando o sinal do halo invertido. Radiol Bras. 2007; 40:355-7.

13. Marques AS. Paracoccidioidomycosis: Epidemiological, clinical and treatment up-date. An Bras Dermatol. 2003;78:135-50. 